\title{
Transverse Single Spin Asymmetries in Electron Scattering on Hydrogen Targets
}

\section{Boxing Gou*}

Helmholtz-Institut Mainz

E-mail: boxingoueuni-mainz.de

\section{Sebastian, Baunack}

Institut für Kernphysik, Johannes Gutenberg-Universität Mainz,

E-mail: baunack@uni-mainz.de

\begin{abstract}
The asymmetry in the scattering of transversely polarized electrons off unpolarized protons or deuterons arises from the imaginary part of the two-photon-exchange amplitude. The A4 collaboration at the MAMI accelerator has performed measurements at various beam energies between $300 \mathrm{MeV}$ and $1.5 \mathrm{GeV}$ both at forward and backward angles. We present an overview about published data and unpublished preliminary results in the elastic scattering and discuss future analyses for inelastic asymmetries in the resonance region.
\end{abstract}

23rd International Spin Physics Symposium - SPIN2018 -

10-14 September, 2018

Ferrara, Italy

*Speaker. 


\section{Introduction}

The measurement of the proton magnetic moment $\mu$ [1] revealed that the proton is a complex object. Since then, study of the proton structure has been a central subject of the nuclear physics. Among various approaches the electron-proton scattering is more efficient due to the fact that the electron is structureless and the electromagnetic coupling constant is small $\left(\alpha_{\mathrm{EM}} \approx 1 / 137\right)$. Small coupling means that multiple-photon-exchange amplitudes are at negligible level as compared to the one-photon-exchange (OPE or Born) amplitude. Therefore it has been regarded as sufficient to interpret electron scattering experiments within the Born approximation. Tuning the energy carried by the exchanged photon (which is quantified with the squared four-momentum transfer $q^{2}=-Q^{2}$ ), the electron scattering can probe the proton structure at different scales. Experiments with large $\mathrm{Q}^{2}$ can study the internal constituents of the proton. The best example is the experimental confirmation of the existence of quarks [2,3]. With small $\mathrm{Q}^{2}$, one can study the electromagnet distributions on the proton surface, which is in general described by 6 form factors $\tilde{F}_{1-6}$ [4]. In the Born approximation these form factors reduce to 2 form factors, which can be parameterized in terms of the electric and magnetic form factors $G_{E}$ and $G_{M}$. Traditionally $G_{E}$ and $G_{M}$ are measured via the Rosenbluth separation method [5]. In 1990s, with the development of the polarization techniques it became possible to access $\mathrm{G}_{\mathrm{E}}$ and $\mathrm{G}_{\mathrm{M}}$ using the polarization transfer method [6, 7]. Surprisingly,

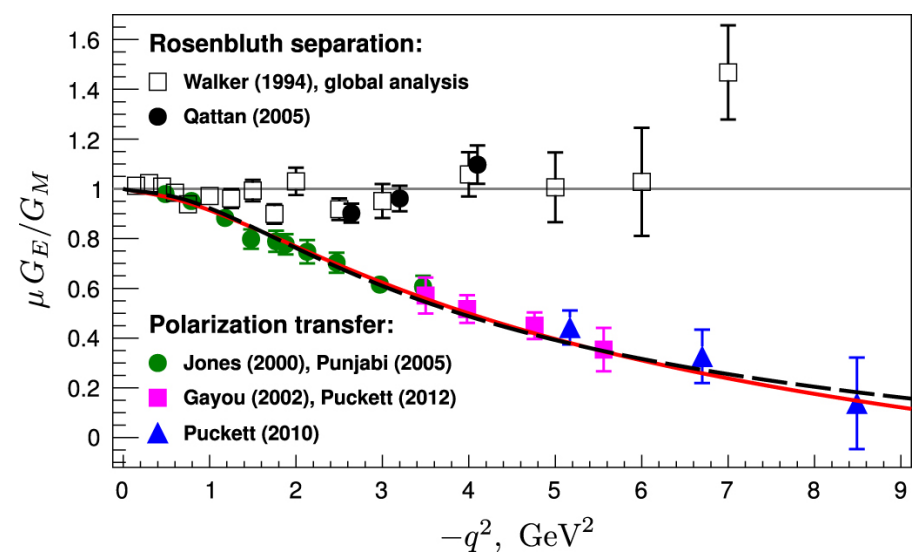

Figure 1: Proton form factor ratio $\mu \mathrm{G}_{\mathrm{E}} / \mathrm{G}_{\mathrm{M}}$ as a function $-\mathrm{q}^{2}$, measured with Rosenbulth separation (black) and polarization transfer (color) methods. The plotted data can be found in Ref. [8] and references therein.

different values of the proton form factor ratio $\mu \mathrm{G}_{\mathrm{E}} / \mathrm{G}_{\mathrm{M}}$ were obtained from these two methods, which is sometimes referred as "proton form factor puzzle". Since both methods are based on the Born approximation, the discrepancy indicates it is necessary to consider processes beyond the one-photon exchange. Indeed it has been shown that it is possible to solve the problem with a two-photon-exchange (TPE) correction [9]. One lesson from the failure of the time-honored Born approximation is that it is crucial to understand how the two-photon exchange would contribute various observables, in order to continuously use the electron scattering as a probe of the hadron structure, especially in high-precision experiments. Observables directly connected with the TPE amplitudes would provide a testing ground for developing a reliable theoretical framework to compute the TPE contributions. The interference of the OPE and TPE amplitudes contribute the elastic scattering cross section with opposite signs, so the ratio between cross sections of electron and 
positron scattering off proton gives access to the real parts of the general form factors $\tilde{\mathrm{F}}_{1-3}$ [10]. Complementarily, the imaginary parts can be investigated via the transverse single spin asymmetry $\mathrm{A}_{\perp}$ in electron-proton scattering [11]. The transverse single spin asymmetry $\mathrm{A}_{\perp}$ is connected to the imaginary parts of the TPE amplitude in terms of $A_{\perp}=\frac{2 M_{1 \gamma} \operatorname{Im}\left(M_{2 \gamma}\right)}{\left|M_{1 \gamma}\right|^{2}}$, with $M_{1 \gamma}$ and $M_{2 \gamma}$ being the OPE and TPE amplitudes respectively. One can measure transverse spin asymmetry with either polarized targets or polarized beams. The target asymmetry provides access to the imaginary parts of $\tilde{\mathrm{F}}_{1-3}$, while the beam asymmetry provides access to $\tilde{\mathrm{F}}_{3-5}$ [4]. Due to the Lorentz contraction, the beam asymmetry is at the order of part per million (ppm) for beam energies around $1 \mathrm{GeV}$. The A4 spectrometer is capable of measuring such small asymmetries. A systematic program on the TPE investigation has been carried out by the A4 Collaboration at MAMI.

\section{Two photon exchange investigation at A4}

The MAMI accelerator [12] consists of a cascade of 4 racetrack microtrons (RTMs), it is able to provide both polarized and unpolarized c.w. electron beams up to $1.6 \mathrm{GeV}$ with an intensity of $20 \mu \mathrm{A}$. The polarized electron source [13] produces longitudinally polarized electrons by illuminating a GaAs crystal with a circularly polarized laser. A Wien filter and a solenoid are installed in the injection beam line, so that the beam spin can be rotated into both the transverse and vertical orientations [14]. To minimize systematic uncertainties arising from possible time instabilities, the beam polarization was flipped every $20.08 \mathrm{~ms}$ by changing the Pockels cell voltage in the laser optics. The spin flipping pattern follows a quadruplet of either $(\uparrow \downarrow \downarrow \uparrow)$ or $(\downarrow \uparrow \uparrow \downarrow)$, randomly set by a bit generator. In this way, any possible false asymmetries associated with slow-drift effects would be suppressed. In addition, a half-wave plate was inserted into the laser for about $50 \%$ of the total data-taking time in order to test and understand any long-term systematic effects. The absolute beam polarization was measured about once per day with a Møller polarimeter located in the A1 hall. The A4 spectrometer is a total absorption electromagnetic calorimeter (EMC) composed of 1022 lead fluoride $\left(\mathrm{PbF}_{2}\right)$ crystals, which are placed symmetrically around the beam axis in 146 alu-
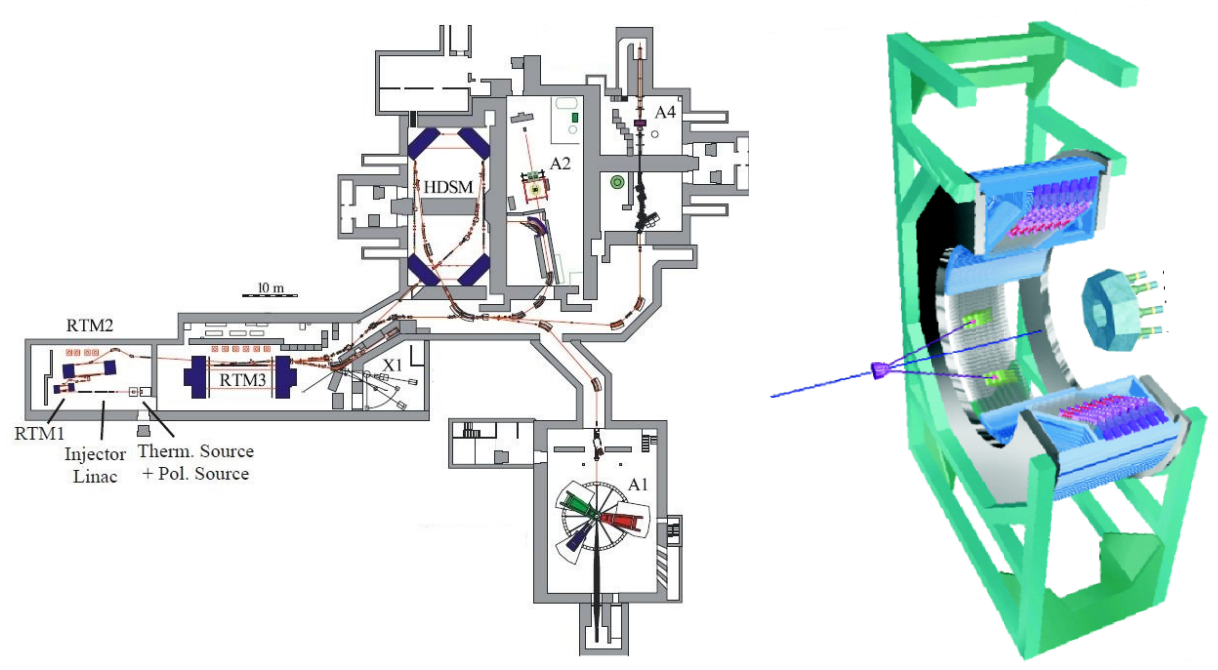

Figure 2: Floor plan of the MAMI accelerator (left) and schematic drawing of the A4 spectrometer (right). 
minum frames. Each frame hosts 7 crystals. The crystals are made in shapes of trapezoidal prisms with a length of $150-180 \mathrm{~mm}$, a front cross section of $26 \times 26 \mathrm{~mm}^{2}$ and a rear cross section of $30 \times 30 \mathrm{~mm}^{2}$. When a particle passes through the EMC, an electromagnetic shower develops over a cluster of $3 \times 3$ crystals. The particle losses more than $95 \%$ of its energy in one cluster in terms of Cherenkov light, which are read out by photomultiplier tubes. To keep the signal gain normalized, the high voltage applied on the photomultipler tubes were adjusted about once per hour based on a realtime calibration procedure [15]. If no shower overlaps occur, the summation of the signals collected in the cluster will be digitized with a 8-bit flash-ADC and stored in a histogramming unit, which is read out and saved on an external storage device for each 5-minute run. The left panel of Fig. 3 shows a typical energy spectrum measured in one run. The event rate of the whole EMC amounts to $100 \mathrm{MHz}$ and the energy resolution for single events is about $3.9 \% / \sqrt{\mathrm{E} / \mathrm{GeV}}$. The EMC is installed on a rotatable platform, so that both forward $\left(30^{\circ}<\theta_{\text {lab }}<40^{\circ}\right)$ and backward $\left(140^{\circ}<\theta_{\text {lab }}<150^{\circ}\right)$ angular acceptances are accessible. In the backward configuration 72 plastic scintillators are placed in front of the $\mathrm{PbF}_{2}$ crystals to discriminate $\gamma_{\mathrm{S}}$ from $\pi^{0}$ decay. A high power cryogenic liquid target system [16] can operate with both hydrogen and deuterium. It features with large target thickness and high density stability. A luminosity monitor (LuMo) is installed downstream, it comprises of 8 water Cherenkov counters around the beam axis and registers electrons emitted at polar angles between $4^{\circ}$ and $10^{\circ}$. The LuMo can be used to measure the target density ratio as well as to monitor the beam polarization.
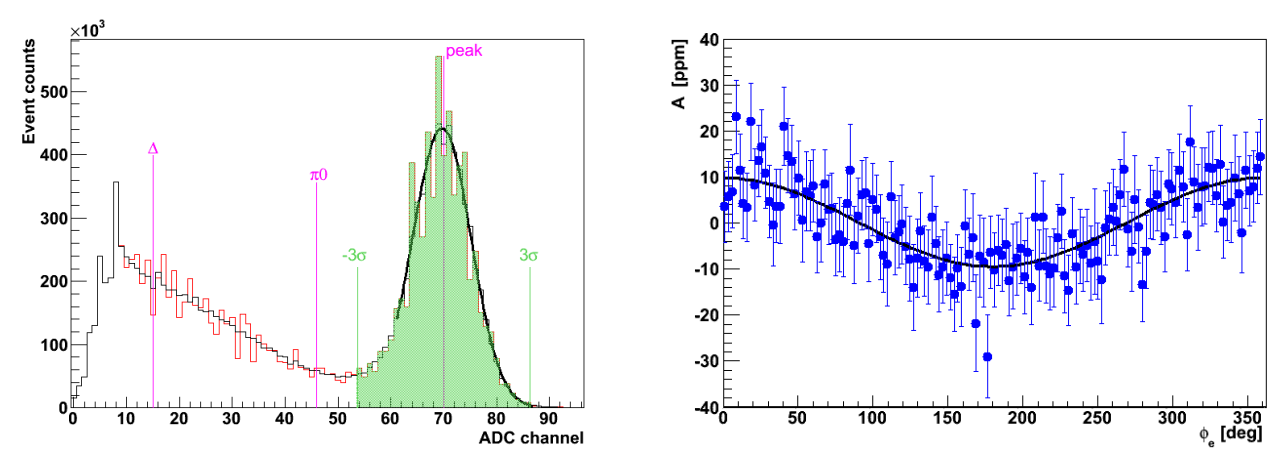

Figure 3: Energy spectrum (left) and azimuthal asymmetry (right) of the scattered elections.

The A4 collaboration has performed measurements of the transverse beam spin asymmetry at various beam energies with hydrogen and deuterium targets at both forward and backward angles. First results of the forward-angle asymmetries at 570 and $855 \mathrm{MeV}$ were reported in 2005 [17]. More recently, the backward-angle asymmetries at 315 and $420 \mathrm{MeV}$ were published [18]. Here we present new forward-angle results at $315,420,510,855$ and $1508 \mathrm{MeV}$. Corresponding to all the combinations of $3 \times 3$ crystal cluster, there are 730 channels in total. For each of the polarization states ( $\uparrow$ and $\downarrow$ ), there is one energy spectrum measured from every channel in every run. The event count of the elastic scattering is determined by an integral over the elastic peak. The integral boundary is chosen such that contamination due to either the inelastic processes or the pile-up events can be neglected. Using the event counts $\left(\mathrm{N}^{\uparrow}\right.$ and $\left.\mathrm{N}^{\downarrow}\right)$ for both of the polarization states, together with the target-density ratio $\mathrm{R}_{\rho}=\frac{\rho^{\uparrow}}{\rho^{\downarrow}}$ which is determined from the LuMo data, a raw asymme- 
try $A_{\text {raw }}=\frac{N^{\uparrow} / R_{\rho}-N^{\downarrow}}{N^{\uparrow} / R_{\rho}+N^{\downarrow}}$ is calculated for every channel. For measurements of asymmetries at ppm level, even small differences in beam conditions between two polarization states would produce false asymmetries comparable to the physics asymmetry. To cope with such false asymmetries, the beam intensity asymmetry $\mathrm{A}_{\mathrm{I}}$, position differences $(\Delta \mathrm{X}, \Delta \mathrm{Y})$, angle differences $\left(\Delta \mathrm{X}^{\prime}, \Delta \mathrm{Y}^{\prime}\right)$ and energy difference $\Delta \mathrm{E}$ between two polarization states are minimized and monitored. In the offline data analyses a correction are applied as $\mathrm{A}_{\text {corr }}=\mathrm{A}_{\text {raw }}-\mathrm{c}_{1} \mathrm{~A}_{\mathrm{I}}-\mathrm{c}_{2} \Delta \mathrm{X}-\mathrm{c}_{3} \Delta \mathrm{Y}-\mathrm{c}_{4} \Delta \mathrm{X}^{\prime}-\mathrm{c}_{5} \Delta \mathrm{Y}^{\prime}-\mathrm{c}_{6} \Delta \mathrm{E}$. The correction coefficients $c_{1-6}$ are determined from a multilinear regression analyses. The right panel of Fig. 3 shows an azimuthal distribution of the weighted average of $\mathrm{A}_{\text {corr }}$ over the channels in the same frame, normalized by the beam polarization. The transverse asymmetry $\mathrm{A}_{\perp}$ is extracted by fitting the distribution with a cosine function. The forward-angle transverse beam spin asym-

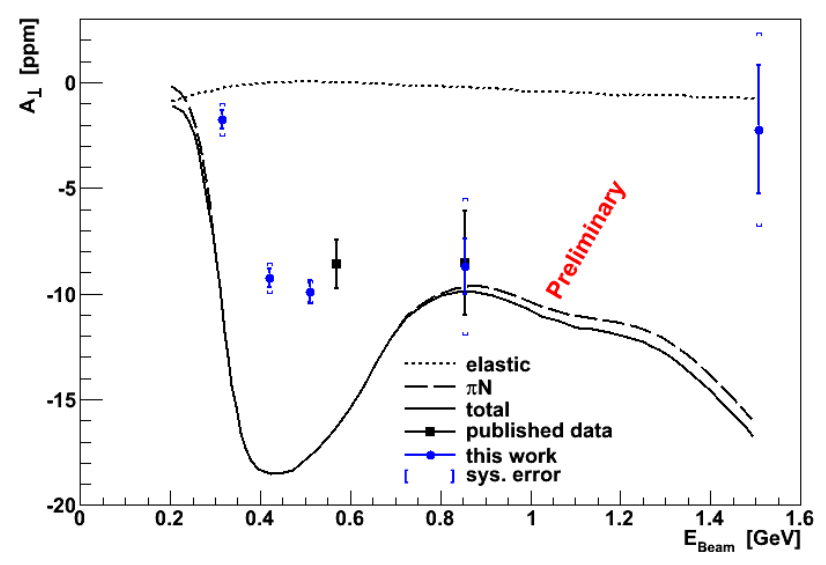

Figure 4: Transverse beam spin asymmetry as a function of the beam energy.

metries measured at A4 are plotted in Fig. 4. The blue dots represent the new measurements. The statistic error are plotted as solid bars; the square brackets indicate the total error including the uncertainties of the regression coefficients. The new measurements are consistent with the previously pudblished data at 570 and $855 \mathrm{MeV}$ [17] (black square). The dotted line and the dashed line are the theoretical calculations based on proton and on $\pi \mathrm{N}$ intermediate states respectively [19]. The calculation that includes both intermediate states are given with solid line. Unlike the backwardangle data [18], which are in gerneral agree with the calculation in Ref. [19], the forward-angle data manifest a different behavior. Although a dip-like structure is also seen around the $\Delta$ production threshold, the asymmetries are about 50\% smaller than the calculation. This indicates that other intermediate states, such as $\pi \pi \mathrm{N}$ states might also be excited. Besides the elastic electron-proton scattering, events of the $\Delta$ production channel are also collected by the A4 EMC (see Fig. 3). Thus the same data provide the opportunity to study the TPE processes in the $\Delta$ electroproduction. We have observed large asymmetries in the inelastic region. Background study is in progress.

\section{Summary and outlook}

To summarize, the proton form factor puzzle has put the Born approximation in electron scattering into question, as a result significant efforts have been made on the two-photon exchange. The transverse single spin asymmetries provide a way to study the TPE amplitudes. A systematic program on the transverse beam spin asymmetries have been carried out by the A4 collaboration. New 
data of the forward-angle asymmetries in the elastic electron-proton scattering have been obtained at 5 energies. A study of the asymmetries of the $\Delta$ production channel is ogonig.

\section{References}

[1] R. Friseh and O. Stern, Über die magnetische Ablenkung von Wasserstoffmolekülen und das magnetische Moment des Protons. I, Z. Physik 85 (1933) 4.

[2] E. D. Bloom et al., High-Energy Inelastic e-p Scattering at $6^{\circ}$ and $10^{\circ}$, Phys. Rev. Lett. 23 (1969) 930.

[3] M. Breidenbach et al., Observed Behavior of Highly Inelastic Electron-Proton Scattering, Phys. Rev. Lett. 23 (1969) 935.

[4] M. Gorchtein et al., Beam normal spin asymmetry in elastic lepton-nucleon scattering, Nucl. Phys. A741 (2004) 234 [arXiv: hep-ph/ 0404206$].$

[5] M. N. Rosenbluth, High Energy Elastic Scattering of Electrons on Protons, Phys. Rev. 79 (1950) 615.

[6] A. I. Akhiezer and M. P. Rekalo, Polarization effects in the scattering of leptons by hadrons, Sov. J. Part. Nucl. 4 (1974) 277.

[7] R. G. Arnold et al., Polarization transfer in elastic electron scattering from nucleons and deuterons, Phys. Rev. C 23 (1981) 363.

[8] A. J. R. Puckett et al., Recoil Polarization Measurements of the Proton Electromagnetic Form Factor Ratio to $Q^{2}=8.5 \mathrm{GeV}^{2}$, Phys. Rev. Lett. 104 (2010) 242301 [arXiv: 1005.3419 ].

[9] P. A. M. Guichon and M. Vanderhaeghen, How to Reconcile the Rosenbluth and the Polarization Transfer Methods in the Measurement of the Proton Form Factors, Phys. Rev. Lett. 91 (2003) 142303 [arXiv: hep-ph/0306007].

[10] M. Rekalo and E. Tomasi-Gustafsson, Model independent properties of two-photon exchange in elastic electron proton scattering, Eur. Phys. J. A 22 (2004) 331 [arXiv: nucl-th/ 0307066 ].

[11] A. De Rújula et al., Elastic scattering of electrons from polarized protons and inelastic electron scattering experiments, Nucl. Phys. $\mathbf{B 3 5}$ (1971) 365.

[12] A. Jankowiak, The Mainz Microtron MAMI - Past and future, Eur. Phys. J. A s01 (2006) 149.

[13] K. Aulenbacher et al., The MAMI source of polarized electrons, Nucl. Instrum. Methods Phys. Res., Sect. A 391 (1997) 498.

[14] B. S. Schlimme et al., Vertical Beam Polarization at MAMI, Nucl. Instrum. Methods Phys. Res., Sect. A $\mathbf{8 5 0}$ (2017) 54 [arXiv: 1612 . 02863].

[15] S. Baunack et al., Real-time calibration of the A4 electromagnetic lead fluoride $\left(\mathrm{PbF}_{2}\right)$ calorimeter, Nucl. Instrum. Methods Phys. Res., Sect. A 640 (2011) 58 [arXiv:1102.5640].

[16] I. Altarev et al., A high power liquid hydrogen target for the Mainz A4 parity violation experiment, Nucl. Instrum. Methods Phys. Res., Sect. A 564 (2006) 13 [arXiv: nucl-ex/ 0504024 ].

[17] F. E. Maas et al., Measurement of the Transverse Beam Spin Asymmetry in Elastic Electron-Proton Scattering and the Inelastic Contribution to the Imaginary Part of the Two-Photon Exchange Amplitude, Phys. Rev. Lett. 94 (2005) 082001 [arXiv: nucl-ex/ 0410013 ].

[18] D. Balaguer Ríos et al., New Measurements of the Beam Normal Spin Asymmetries at Large Backward Angles with Hydrogen and Deuterium Targets, Phys. Rev. Lett. 119 (2017) 012501.

[19] B. Pasquini and M. Vanderhaeghen, Resonance estimates for single spin asymmetries in elastic electron-nucleon scattering, Phys. Rev. C 70 (2004) 045206 [arXiv: hep-ph / 0405303 ]. 